Témoigner Témoigner. Entre histoire et mémoire

Getuigen Revue pluridisciplinaire de la Fondation Auschwitz

$126 \mid 2018$

Questions sur l'avenir du travail de mémoire

\title{
La maison de l'histoire européenne : un musée qui pose (des) question(s)
}

Het huis van de Europese geschiedenis: een museum waar(over)vragen gesteld worden

\section{Christine Dupont}

\section{(2) OpenEdition}

\section{Journals}

Édition électronique

URL : https://journals.openedition.org/temoigner/6885

DOI : $10.4000 /$ temoigner.6885

ISSN : 2506-6390

Traduction(s) :

Het Huis van de Europese geschiedenis: een museum waar(over) vragen gesteld worden - URL

https://journals.openedition.org/temoigner/6930 [nl]

Éditeur :

Éditions du Centre d'études et de documentation Mémoire d'Auschwitz, Éditions Kimé

Édition imprimée

Date de publication : 2 avril 2018

Pagination : 10-13

ISBN : 978-2-930953-06-9

ISSN : 2037-4183

Référence électronique

Christine Dupont, «La maison de l'histoire européenne : un musée qui pose (des) question(s) », Témoigner. Entre histoire et mémoire [En ligne], 126 | 2018, mis en ligne le 24 janvier 2022, consulté le 04 février 2022. URL : http://journals.openedition.org/temoigner/6885 ; DOI : https://doi.org/10.4000/ temoigner.6885 


\section{LA MAISON DE L'HISTOIRE EUROPÉENNE : UN MUSÉE QUI POSE (DES) QUESTION(S)}

EXPOSITION «Un musée qui pose plus de questions quil n'apporte de réponses. » Voilà l'une des nombreuses réactions suscitées par la Maison de l'histoire européenne depuis son ouverture en mai 2017. Plusieurs visiteurs nous disent qu'ils śattendaient à un discours de propagande européenne (le musée fait partie du Parlement européen, l'entrée est gratuite). Certains sont surpris de découvrir une histoire plus nuancée et plus critique. Dautres sont étonnés, parfois fâchés de nos choix. II semble bien que le musée laisse peu de visiteurs indifférents.

METTRE EN MUSÉE L'HISTOIRE DE LEUROPE

e relatifétonnement des visiteurs répond en réalité parfaitement à la difficulté de la tâch qui s'ouvrait devant nous lorsque, en jan ier 2011, nous avons commencé à réfléchi à la forme qu'un musée d'histoire de l'Europe pouvait evêtir.Ce «nous» désigne une équipe scientifique d'hisentre entre personnes de díententes générations, issues de de la complémentarité d'expériences très diversison de la complémentarité dexpériences très diverses de en milieu muséal Cette diversité se reflète, aux dires en milieu muséal. Cette divesír se reflete, aux dire dont les contenus de notre musée sor trús.

Mettre en récit l'histoire de l'Burope dans un

Mettre en récit l'histoire de l'Europe dans un l'Union Union eir déf à relever. Partir d'une page blanection, téćtaitle

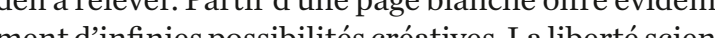
ifiqu totale que nous a laissée le Par. La libertéscieninitiat to inter en importants nis à sá disposition, ontétédes atoutsnon et pouvoir fincent ouvir les portes du mú et pouvoir finalement ouvrir les portes du musée le
Les seules lignes directrices existantes, jetées sur apier dès 2008 par un comité d'experts, mettaient bien sûr l'accent sur l'histoire de la construction européenne et sur l'histoire du XXe siècle. Pour le reste, nous étions libres de développer les contenus du futur musée selon nos propres approches. Un travail fascinant de conceptualisation, de négociations, d'écriture de recherche d'objets. Une expérience dontj'aimeà dire qu'elle a modifié en profondeur ma vision de l'Europe, et même mon rapport à l'histoire.

\section{QU'EST-CE QUE L'EUROPE ?}

Le choix de faire un musée d'histoire s'est d'abord raduit par le développement d'une trame narrative. Nous pom le développenent dune trane narrative. d'événem devénents éc de concepts que nous avons idenliens connle nés en Europe, s'étant dóveloppés sur lensenbéritage est toujours bien présu continent et dont héritage est toujours bien présent aujourd hui.

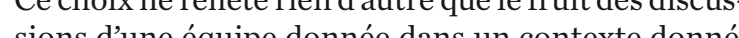
sions d une équipe donnée dans un contexte donné. Unent choisi d'autres options. mentlaprétention d'offrir un récit définitif l'histoire de l'Europe.

L'histoire qui constitue la dorsale de notre exposition permanente commence par une question
Qu'est-ce que l'Europe? Qu'il s'agisse du mythe de cette princesse phénicienne enlevée par Zeus transform en taureau ou de l'étendue géographique du continen (quelles sont les frontières orientales de l'Europe ?) l'objectif est d'introduire d'emblée toute la complexité d'une histoire qui ne va pas de soi. Nous compléton cette introduction par une réflexion autour de quatorze éléments de base qui représentent notre proposition de ce que pourrait être l'héritage du passé purón l' l'e l'omnésésento du

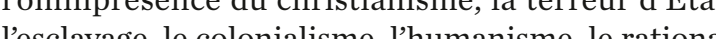
liscave, le coloniansme, lhumanisme, le rationale le génocide.

Ces thèmes se retrouvent ensute à travers le Ces thèmes se retrouvent ensuite à travers découlé de lexposition permane, qui, dès la fin de tour chrono $A$

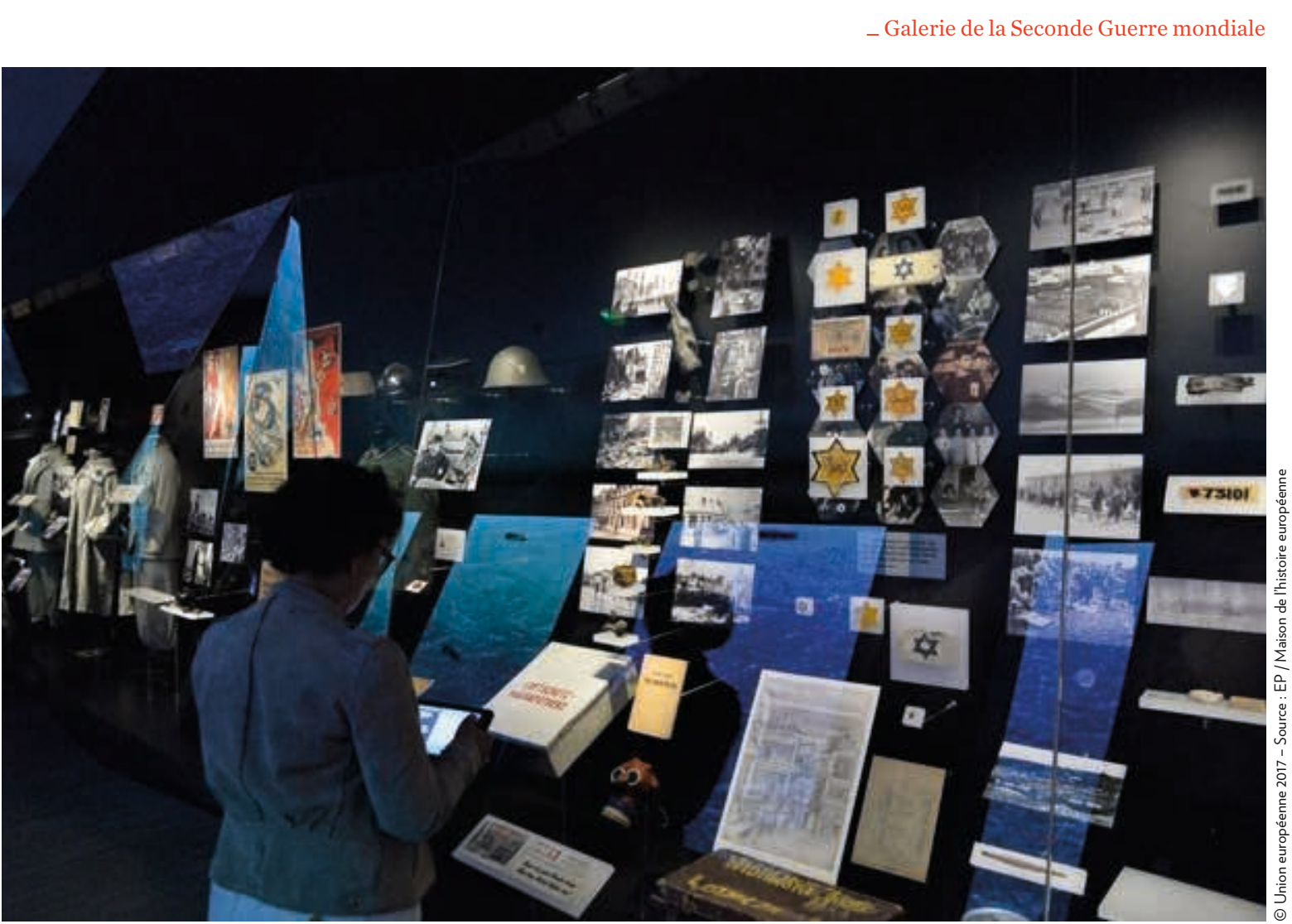

caise, moment fondateur de notre système politique moderne, jusqu'à aujourd'hui (le Brexit n'a pas été occulté), le fil d'une histoire du continent européen e déroule selon des choix thématiques qui privilégien toujours une approche transnationale plutôt qưune ddition d'histoires nationales.

La décision de faire un «vrai» musée avec des objet originaux a bien entendu orienté nombre de choix. Labsence d'une collection nous a permis de construire une très riche collaboration avec plus de 300 musée pountiellement européens qui nous prêtent, souvent

LA PLACE CENTRALE DE LA SHOAH

Il serait trop long et fastidieux de présenter ici une sorte de visite virtuelle de notre exposition permanente.Du reste, le medium exposition ne s'appréhende

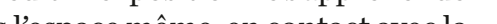

Galerie de la Seconde Guerre mondial pour plusieurs années, des objets de leurs collections. 
•. scénographie, les objets, les dispositifs de médiation. Je ne prendrai qu’ exemple, notamment parce quil semble déconcerte certains visiteurs. Il sagt de la manière dont nous la Shoah.

Au lendemain de sa visite lors de l'ouverture pour la presse, le jourKuczkiewicz titrait son Kuczkiewicz titrait son article « La Maison d histoire européenne: un musée où il manque un gros "détail". » mot de Jean-Marie mot de Jean-Marie Le Pen de sinistre memoire, il y critiquait vivement notre presentation de la Shoah, regretsalle spécifiquement consacréeà ce thème. Manifré à ce thème. Manifestement, ce choix étonne et peut
déconcerter.

Pourtant, la Shoah ccupe une place centrale dans l'ensemble de notre dans lensemble de notre exposition permanente. tion (vês cè lintroduction (voir ci-dessus), le genocide des Juifs d Europe apparaît à de nombreuses reprises tout au longde l'exposition. Dan la partie surleXIXe siècle, loppées en Europe sont (1) Le Soir Plus, 6 mai 2017,
aticle publié également dans article publié également dans le journal papier

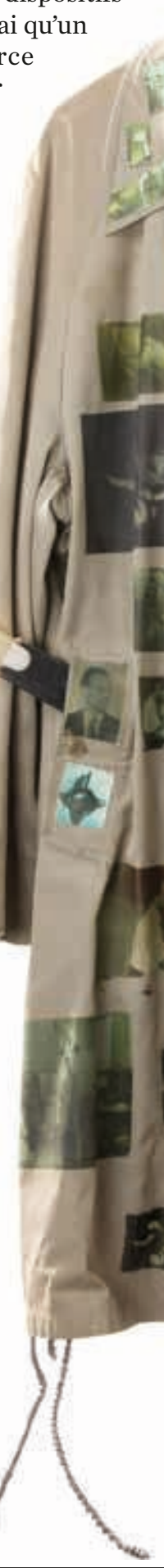

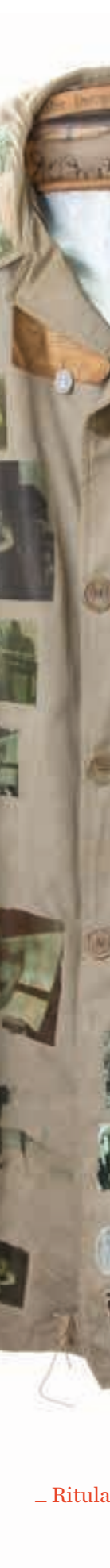

西 dans un souci de contextualiser de loppement large leur développement au siècle suivant par le régime nan. par le régime nazi.

L’annihilation planifiée des Juifs d'Europe apparaît ensuite comme focale principale de ce régime dans la partie de l'exposition qui compare (sans un pied d'égar auté) lest sur un pied degalité) les deux principales dictature chapitre « géno-1940. Le chapitre « genocide et terreur de masse » y présente entre autres objets une boite de Zyklon B (Musée L'Appel : L'Appel : un dessin d'Ella 1998) qui ́́vile (19271998) qui évoque le terd'extermintion (Ghetto Fighters'House (Ghetto Isräl) Israèl), ou encore une copie des plans d'un $\mathrm{Kre}$ (YadVashem, Birkenau La galerie suivante est majoritairement occupée par une vaste vitrine aux lignes ture pan-européenne lecture pan-européenne de la Seconde Guerre mondiale. Laplus grande partie de ce disfrances des populatioux souf(la guerre e totalions civiles (la guerre « totale »), partout but plus lourd avec un tribut plus lourd encore payé par le centre et l'est du continus, les exécutions des rete$\begin{aligned} \text { les daränkel et Nicholas Morris, } & \text { nus, les exécutions de masse, } \\ \text { Le manteau de Josef, } 2001 & \text { les déportations, les camps de }\end{aligned}$ concentration et bien entendu la Shoah en tant que telle présentent un grand nombre d'exemples directement liés à la persécution et à l'extermination des Juifs en Europe. Parmi quelques histoires choisies de résistance, le sauvetage des Juifs apparaît également, (anters. Dans l'espace qui conclut an quelque sorte cette période noire de la

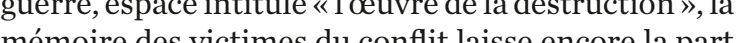
mémoire des victimes du confte lasse encore la part elt d'alletions est daalleurs présentée de manière discursive par distantes de quarante ans, celle de Winsu parce que) uisolite un acte beni de l'o Whis (1946) et celle qui sole detle d'Elie Wiesel qui en appelle à la mémoire pour «sauv
l'humanité » (1986).

Cette dernière citation fait référence à la mémoire de la Shoah que nous avons voulu présenter en tant de la Shoah que nous avons voulu présenter en tant que telle dans un espace separe, a létage suivant de lexposition, soit dans la tranche chronologique des années 1945-1975. Unchoix conplexe que nous assumons pleinemén idéologies et leur brutale mise en œuvre de mons idéologies et ten britale nise sien contextualisée au sein de differentes thématiques rappeler l' problém ticue de sa mémoire. Lo dispositif ćactère problénatique de sá ón phique est dépouille. Dans le fond de la salle, louvre dosef interroge la question du souv, le Manteau de Josef, interroge la question du souvenir à travers ce präntrel. Toute une série de souvenirs le ce surve, Josef de la Sho sont cousus à în l'étofe du mura de la Shoah sont cousus à mêne létoffe du manteau. De part et dautre se trouvent deux grandes vitrines, présentant chacune troisécudes de cas sur la manière do quent la question deless eur exemples choisis sont les deux Allemagnes, lAutriche, la Pologne, la France et l'Ukraine. Certains débats sont toujours en cours et montrent bien a quel point la recon alssance de ces chir lis sémoire erédent est au cœur des discussions surio nén l'his ouropénne, nique Tony Judt qui figure nique Tony Judt qui figure en exergue de cette salle: «La ménoirétrouvée des Juifs morts de leuropeest de l'humanité restaurée du continent. »
UNE HISTOIRE TROP NÉGATIVE ?

Certains nous reprochent d'avoir trop mis l'accent cer le passé traumatique de l’Europe. Cette vision ne rend pas justice a la richesse de l'exposition pernanente qui est loin de se limiter aux heures noires du $x^{e}$ eicle. Elle ignore ausi la premiere exposition (x) péens, par-delà les frontières et les époques. De manièréććla les frograè poraires st précíment pensé pourêtrecomb́ntaire delexposition permanente, appostecon lus cuturel sur le passé europén tabordéc périodes de l'histoire.

Mais le débat n’est pas clos. Je dirais même qu'il ne ait que conmencer Concevoir, réaliser et faire vivre an muse d' histoire de l'Europe dans le contexte qu évidura dourd hui n'est évidemment pas une sinécure. Conscients davoir fait la discussion.

Christine Dupon Conservatrice

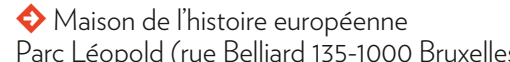

https://historia-europa.ep.eu

Entrée gratuite Exposition « Interactions. Des siècles de commerce, de combat
et de création » jusqu'au 31 mai 2018. 